\title{
Nanoscale
}

Check for updates

Cite this: Nanoscale, 2019, 11, 2878

\section{Polyelectrolyte multilayers of poly (L-lysine) and hyaluronic acid on nanostructured surfaces affect stem cell response $\uparrow$}

\author{
Marcus S. Niepel, (DD a Bhavya K. Ekambaram, ${ }^{b}$ Christian E. H. Schmelzer ${ }^{c}$ and \\ Thomas Groth (iD *d
}

\begin{abstract}
Laser interference lithography (LIL) and the layer-by-layer (LbL) technique are combined here for the first time to design a system with variable nanotopographies and surface viscoelasticity to regulate cell behavior. LIL is used to generate hexagonally arranged nanostructures of gold with different periodicity. In contrast, LBL is used to assemble a multilayer system of poly-L-lysine and hyaluronic acid on top of the nanostructures. Moreover, the viscoelastic properties of that system are controlled by chemical cross-linking. We show that the topography designed with LIL is still present after multilayer deposition and that the formation of the multilayer system renders the surfaces hydrophilic, which is opposite to the hydrophobic nature of pristine nanostructures. The heterogenic system is applied to study the effect on adhesion and differentiation of human adipose-derived stem cells (hADSC). We show that hADSC spreading is increasing with cross-linking degree on flat multilayers, while it is decreasing on nanostructures modified with multilayers. In addition, early effects on signal transduction processes are seen. Finally, hADSC differentiation into chondrogenic and osteogenic lineages is superior to adipogenic lineages on nanostructures modified with multilayers. Hence, the presented system offers great potential to guide stem cell differentiation on surfaces of implants and tissue engineering scaffolds.
\end{abstract}

Received 9th July 2018, Accepted 10th December 2018

DOI: $10.1039 / \mathrm{c} 8 \mathrm{nr} 05529 \mathrm{~g}$

rsc.li/nanoscale vival are controlled by integrin ligation, focal adhesion (FA) formation, and subsequent signal transduction processes. ${ }^{4}$ Not only the presence of these chemical cues, but also their spacing is a decisive factor. ${ }^{5}$ Moreover, the mechanical properties of the ECM have a strong impact on cell behavior. ${ }^{6,7}$ As a result, biomaterials with a specific nanotopography as well as chemical composition could be used to regulate cellular behavior by controlling the organization of cell adhesive structures, cytoskeletal arrangement and tension and, thus, outside-in signaling processes..$^{8-11}$

Nanofabrication methods can be classified into top-down and bottom-up approaches. ${ }^{12,13}$ While with the latter higher ordered structures are formed by self-assembly or self-organization of small entities, the former apply different techniques to remove material from larger entities to form structures of interest. Typical examples for top-down approaches include microcontact printing $(\mu \mathrm{CP}),{ }^{14,15}$ electron beam lithography $(\mathrm{EBL}){ }^{16}$ photolithography, ${ }^{17}$ or nanoimprint lithography (NIL), ${ }^{18}$ which are mostly cost-intensive with a relatively low throughput on small patterning areas. In contrast, typical bottom-up methods are phase segregation of silanes, colloidal gold lithography, polymer demixing, and other self-assembly procedures such as the layer-by-layer (LBL) technique to design structures in the sub-micron or even low-nanometer 
range. ${ }^{12,19-21}$ In addition, combinations of both approaches are possible. The nanosphere lithography (NSL), for example, combines colloidal template formation (bottom-up) with noble metal deposition (top-down). ${ }^{22,23}$ However, the high sample-tosample variance regarding the long-range order due to the self-assembly of the mask is a clear drawback of this technique. ${ }^{22}$ Nevertheless, it is capable to control adhesion and growth of tissue cells. ${ }^{22}$ In a recent work we showed that the nanofeature dimensions obtained by NSL can be generated defect-free by interferometric lithography, but with improved long-range order. ${ }^{24}$ This technique is called laser interference lithography (LIL) and its benefit is the cost-efficient and fast patterning of surfaces at nanoscale over large areas. ${ }^{25,26}$ There, the interference of mutually coherent light beams leads to periodic and quasi-periodic interference patterns on a photosensitive material. With this mask-less technique, the period $p$ of the interference pattern can be adjusted with the wavelength $\lambda$ of the light source together with a change of the angle of incidence. ${ }^{27}$ Moreover, one-dimensional regular patterns or twodimensional structures, for example hexagonally arranged dots, can be generated by two-beam or three-beam LIL. ${ }^{27,28}$

The LbL technique as a classical bottom-up technique has emerged recently as widely used method to design material coatings with different molecules, mostly polyelectrolytes (PEL). ${ }^{29}$ With this technique, chemical cues can be introduced to biomaterial surfaces that address cell receptors. ${ }^{30,31}$ Moreover, parameters like wetting properties, surface potential, topography, thickness, and viscoelasticity can be regulated by selection of PEL and by control of process parameters during multilayer formation. ${ }^{31-33}$ In case proteins and glycosaminoglycans (GAG) were used for multilayer assembly, the structure and composition of ECM could be mimicked. ${ }^{33,34}$ Moreover, the rather high water content of such multilayer systems offers specific viscoelastic properties that can be tailored either by the complexation conditions or by chemical cross-linking. ${ }^{35,36}$

In previous studies we could show that LIL can be used to design defect-free structures of gold of different size and periodicity on silicon as model surface. ${ }^{24}$ We could demonstrate further that spreading of human adipose-derived stem cells (hADSC) decreased with increasing feature dimensions, which had an effect on cell differentiation. ${ }^{24}$ In another study we studied how polyelectrolyte multilayers (PEM) made of hyaluronic acid (HA) and poly-L-lysine (PLL), either as native or cross-linked system varying in elastic modulus, guide hADSC adhesion, growth, and differentiation. ${ }^{37}$ We found that hADSC spread and grew more on stiffer, highly cross-linked than on soft native PLL/HA multilayers. It was also observed that hADSC moved towards the osteogenic lineage on stiffer and to adipogenic on soft, non-cross-linked multilayers. Here, we were interested to combine both techniques to create a system that connects topography at the nanoscale produced by LIL with viscoelastic cues. Hence, we prepared nanopatterned surfaces of gold on silicon of three different sizes and periodicities, which were subsequently coated with multilayers composed of PLL and HA with different degree of chemical cross- linking. ${ }^{37,38}$ The novelty of the study is the combination of a technique that produces nanostructured surfaces of low sample-to-sample variation (LIL) with a technique that allows the deposition of molecules affecting overall surface properties (LbL). Especially the use of spray-assisted coating of the structured surfaces is reported here for the first time. Hence, a unique system of topographical and viscoelastic cues is generated to study the effect on hADSC adhesion, signal transduction, and cell differentiation. Results are reported herein.

\section{Experimental section}

\section{Surface cleaning}

Silicon wafers (Si-Mat, Germany) as model surfaces for PEM formation were cleaned before use with 'RCA clean 1' as previously described. ${ }^{37}$ Briefly, ultrapure water and ammonia (25\%, Roth, Germany) were mixed and heated to $75-80{ }^{\circ} \mathrm{C}$, and hydrogen peroxide (35\%, Roth) was added to achieve a ratio of $5: 1: 1(\mathrm{v} / \mathrm{v} / \mathrm{v})$. After $10 \mathrm{~min}$ incubation, the samples were excessively rinsed with ultrapure water ( 6 cycles of $5 \mathrm{~min}$ ), dried with a stream of nitrogen, and used immediately.

\section{Laser interference lithography (LIL)}

Hexagonally arranged nanostructures were designed by threebeam LIL as previously described. ${ }^{24}$ Briefly, silicon surfaces were cleaned with acetone (Roth) and isopropanol (Roth) before use and cut to $(20 \times 20) \mathrm{mm}^{2}$. An adhesion promoter and, subsequently, a negative photo resist (All Resist, Germany) were spin-coated to the cleaned silicon samples and baked at different temperatures. After cutting the samples to $(10 \times 10) \mathrm{mm}^{2}$, they were illuminated with a UV laser (FQCW266-10, Crylas GmbH, Germany, $\lambda=266 \mathrm{~nm}$ ) at different angles of incidence $\left(14^{\circ}, 20^{\circ}\right.$, and $\left.36^{\circ}\right)$ to obtain different feature dimensions. After a post-baking and developing process, the samples were rinsed with copious amounts of ultrapure water, dried, and mounted in a vacuum chamber for metal deposition. All samples were coated with $10 \mathrm{~nm}$ chromium and $50 \mathrm{~nm}$ gold using electron beam physical vapor deposition (EBPVD, FeroTec, Germany). Finally, the photoresist was lifted-off with a sonication sequence in acetone (Roth), ethanol p.a. (Roth), and ultrapure water to obtain discrete nanostructures. All samples were dried with a stream of nitrogen and stored in a desiccator until further use.

\section{Self-assembled monolayer formation}

SAM were used to passivate and activate the nanostructured samples with varying surface chemistry as previously described. ${ }^{24}$ Briefly, a long-chain organo-silane with 43 poly (ethylene glycol) (PEG) units was synthesized and linked to the silicon background. The nanostructured samples were immersed in a $0.25 \mathrm{mM}$ solution of PEG-silane in dry $99.5 \%$ toluene (Roth) and $1 \mu \mathrm{L}$ of triethylamine (Sigma-Aldrich, Germany) was added. The samples were incubated at $80{ }^{\circ} \mathrm{C}$ under nitrogen atmosphere overnight $(18 \mathrm{~h})$ and afterwards sonicated with $99.5 \%$ pure ethyl acetate (Roth) for $2 \mathrm{~min}$, 
rinsed with ethyl acetate and methanol, and dried with a stream of nitrogen. After passivation, the gold nanostructures were activated by adding terminal carboxyl groups using thiol chemistry and, thus, providing a negative net charge. For this reason, all samples were incubated in a $2 \mathrm{mM}$ solution of mercaptoundecanoic acid (MUDA, Sigma-Aldrich) in 99\% ethanol (Roth) at room temperature (RT) overnight. After $18 \mathrm{~h}$ of incubation, the samples were rinsed twice with the solvent as well as ultrapure water, dried with a stream of nitrogen, and stored in a desiccator until further use.

\section{Polyelectrolyte multilayer formation}

A PEM system was assembled using poly (ethylene imine) (PEI, $750 \mathrm{kDa}$, Sigma-Aldrich) as primary layer as well as hyaluronic acid (HA, $301 \mathrm{kDa}-450 \mathrm{kDa}$, Lifecore Biomedical, USA) as polyanion and poly-L-lysine (PLL, $\sim 52 \mathrm{kDa}$, Alamanda Polymers, USA) as polycation. HA and PLL were dissolved at a concentration of $0.5 \mathrm{mg} \mathrm{mL}^{-1}$ in $0.15 \mathrm{M}$ sodium chloride solution while PEI was dissolved at $2 \mathrm{mg} \mathrm{mL}^{-1}$ in the same solution. The $\mathrm{pH}$ of all solutions was adjusted to 6.0. A total number of 24 layers with HA as terminal layer were assembled and abbreviated as [PEI-(HA-PLL) ${ }_{11}-\mathrm{HA}$ ], which means that 11 bilayers of (PLL/HA) were sandwiched by a basal PEI and a terminal HA layer. Flat PEM systems (F-PEM) were prepared by dip coating, while the PEM systems on nanostructured samples (S-PEM) were achieved by a spray coating process. In case of dip coating, all samples were first dipped into the PEI solution at RT for 30 min while gently shaking. Thereafter, the samples were alternately coated with HA and PLL for $15 \mathrm{~min}$ each. Excess PEL solution was removed by rinsing with the solvent twice after each layer for $2.5 \mathrm{~min}$. Finally, all samples were dipped into ultrapure water at $\mathrm{pH}$ 6.0, dried with a stream of nitrogen, and stored in a desiccator until use. In case of spray coating, the nanostructured samples were first dipped into the PEI solution at RT for $30 \mathrm{~min}$. Thereafter, all samples were rinsed with $0.15 \mathrm{M}$ sodium chloride and ultrapure water, dried with a stream of nitrogen and mounted to a sample holder with double-sided tape to continue with the deposition steps using a spray-coater (ND-SP Spray Coater 11/4 $\mathrm{H}$, Nadetech Innovations S.L., Spain). Each deposition cycle consists of adsorption of HA, followed by a rinse with sodium chloride solution, the adsorption of PLL and final rinse step with chloride solution. Thereby, a flowrate of $100 \mathrm{~mL} \mathrm{~h}^{-1}$, a speed of $1 \mathrm{~m} \mathrm{~min}^{-1}$ and 12 steps per layer were used to spray the solutions in a vertical manner $40 \mathrm{~mm}$ away from the surface, while the samples were mounted on a sample holder with a tilt angle of $30^{\circ}$. The spray coating was repeated until 24 layers were achieved, with HA as the terminal layer.

\section{Cross-linking of polyelectrolyte multilayers}

The mechanical properties of the assembled PEM were controlled by chemical cross-linking after the LbL process. It is based on the reaction of activated carboxylic acid groups with primary amino groups in the presence of a water-soluble crosslinker. For film cross-linking, 1-ethyl-3-(3-dimethylaminopropyl) carbodiimide hydrochloride (EDC, Alfa Aesar,
Germany) was dissolved in $0.15 \mathrm{M}$ sodium chloride at $\mathrm{pH} 5.0$ to obtain three different final concentrations $\left(2 \mathrm{mg} \mathrm{mL}^{-1}\right.$, $10 \mathrm{mg} \mathrm{mL} \mathrm{m}^{-1}, 50 \mathrm{mg} \mathrm{mL}{ }^{-1}$ ). $N$-Hydroxysuccinimide (NHS, Sigma-Aldrich) was dissolved in the EDC solutions at a concentration of $11 \mathrm{mg} \mathrm{mL}^{-1}$ to improve the efficiency of the reaction. All samples were incubated with the prepared EDC-NHS solution at $4{ }^{\circ} \mathrm{C}$ under constant shaking for $18 \mathrm{~h}$. Afterwards, the samples were rinsed thrice for $1 \mathrm{~h}$ using $0.15 \mathrm{M}$ sodium chloride at $\mathrm{pH}$ 8.0, dipped into ultrapure water, dried with a stream of nitrogen, and stored until further use.

\section{Surface morphology}

The distribution and morphology of PEM-modified nanostructures was investigated with atomic force microscopy (AFM, Nano-R, Pacific Nanotechnology, CA, USA) and scanning electron microscopy (SEM, ESEM XL 30 FEG, Philips, The Netherlands). The AFM was used in close-contact mode and all scans where performed in dry state with air as ambience. At least two samples were investigated per condition to measure the height and periodicity of nanostructures. Further, the SEM was used in high vacuum mode $\left(p=10^{-6} \mathrm{mbar}\right)$ to determine nanostructure dimensions. Image analysis was performed using the software Gwyddion (version 2.40).

\section{Surface wettability}

The wetting properties of S-PEM as well as F-PEM were characterized with an OCA 15+ system (DataPhysics Instruments, Germany). Static water contact angles (WCA) were measured by adding droplets of fresh ultrapure water $(V=1 \mu \mathrm{L})$ to each surface. Further, dynamic WCA were recorded dispensing $5 \mu \mathrm{L}$ ultrapure water with a velocity of $0.2 \mu \mathrm{L} \mathrm{s}^{-1}$ to the surface and aspirating it with the same velocity. Overall, three samples of each condition of two independent experiments were dimensioned dispensing four to five droplets on each sample. Dynamic WCA $<10^{\circ}$ were considered as zero due to the inappropriate signal-to-noise ratio.

\section{Cell adhesion studies}

Human adipose-derived stem cells (hADSC, Lonza, Switzerland) were used in this study to investigate the influence of nanostructure size and distance in combination with a PEM system on various cellular processes. ${ }^{24}$ Briefly, hADSC were cultured under serum-free conditions using SPE IV medium (ABCellBio, France) in culture flasks, which have been pre-coated with $0.55 \mu \mathrm{g} \mathrm{cm}^{-2}$ human fibronectin (FN, $1 \mathrm{mg} \mathrm{mL}{ }^{-1}$, YO Proteins, Sweden) and $0.50 \mu \mathrm{g} \mathrm{cm}^{-2}$ human collagen type I-III (200 $\mu \mathrm{g} \mathrm{mL}^{-1}$, ABCellBio) in $\alpha$-MEM medium (Gibco, ThermoFisher Scientific, Germany). At 80\% confluency, the cells were harvested with $0.05 \%$ trypsin/0.02\% EDTA solution (Biochrom, Germany) at $37{ }^{\circ} \mathrm{C}$ for 3-5 min. The trypsin reaction was stopped by adding cell growth medium ( $\alpha$-MEM with $10 \%$ fetal bovine serum (FBS, Biochrom) and 1\% antibiotics). After centrifugation at $250 \mathrm{~g}$ for $5 \mathrm{~min}$, the cells were re-suspended in cell growth medium and seeded at a density of 25000 cells per mL on PEM-modified nanostructures. In contrast, flat PEM were pre-coated with $0.50 \mu \mathrm{g} \mathrm{cm}^{-2}$ human col- 
lagen type I-III at $37^{\circ} \mathrm{C}$ for $1 \mathrm{~h}$. Hence, hADSC were seeded in medium without serum on theses surfaces. However, cell adhesion was investigated after $4 \mathrm{~h}$ and $24 \mathrm{~h}$ in both cases and the cellular structures were visualized with immunocytochemistry. Details of the staining protocol can be found in the ESI. $\dagger$ Briefly, adherent and fixed hADSC were permeabilized and non-specific binding sites were blocked with bovine serum albumin solution (BSA, Roth). Thereafter, the actin cytoskeleton, the nucleus as well as vinculin in focal adhesion complexes were visualized. Simultaneously, cells in another set of samples were stained for the small GTPase Ras homolog family member A (RhoA) as well as the focal adhesion kinase (FAK). Finally, all samples were mounted to object slides and examined with confocal laser scanning microscopy (CLSM, LSM710, Zeiss, Germany). At least five images per samples (10×, 20× objectives) were used to determine cell count and morphology using Zeiss efficient navigation (ZEN 2012) and ImageJ (version $1.49 \mathrm{~m}$ ) software.

\section{Cell differentiation}

Human ADSC seeded on S-PEM as well as F-PEM surfaces were also investigated for adipogenic, chondrogenic, and osteogenic differentiation using immunocytochemistry by targeting proteins specific for the lineage development. Briefly, hADSC were seeded at a density of 25000 cells per sample per well in cell growth medium and cultured for seven days at $37^{\circ} \mathrm{C}$ to obtain a certain degree of confluency. Thereafter, the medium was replaced by adipogenic, chondrogenic, and osteogenic induction media of compositions listed in Table S1 in the ESI. $\dagger$ Cells were then incubated for 21 days, while the media were changed twice per week. Finally, the cells were stained for specific differentiation markers for of adipogenic (perilipin and GLUT4), chondrogenic (collagen II and aggrecan), and osteogenic (collagen I and osteocalcin) differentiation. Details of the staining protocol can be found in the ESI. $\dagger$

\section{Statistical analysis}

All results were statistically analyzed by calculating mean and standard deviation. The significance of the means was calculated by one-way analysis of variance (ANOVA) followed by posthoc Tukey's test and the significance level of $p<0.05$ is indicated in the respective figures.

\section{Results}

\section{Nanostructure formation and chemical surface modification}

Nanostructures of gold were generated by LIL, as previously described..$^{24}$ Three angles of incidence $\left(14^{\circ}, 20^{\circ}\right.$, and $\left.36^{\circ}\right)$ were used to generate structures with a periodicity of $733 \mathrm{~nm}$ (large, L), $518 \mathrm{~nm}$ (medium, M), or $302 \mathrm{~nm}$ (small, S). Thereby, the practical values fully met the theoretically predicted values. ${ }^{24}$ After passivation of the substrate with a long-chain PEG and activation of the gold nanostructures with a terminal carboxyl group using MUDA as shown previously, ${ }^{24}$ a PEM system consisting of PLL and HA was sprayed on top of the nano- structures until 24 layers were obtained with HA as terminal layer. AFM measurements performed in dry state with air as ambience proofed the decrease in periodicity by increasing the angle of incidence during nanostructure formation (Fig. 1). In addition, the height profiles obviously changed due to the multilayer coating. Here, the total height dropped with decreasing nanostructure dimension. However, this decrease was not seen on unmodified nanostructured surfaces, which had a similar height on all dimensions (Fig. S1†). Nevertheless, a homogenous distribution of the deposited biomolecules was seen on the surface since no agglomerates were found on the scanned areas, which was additionally proven by SEM investigations (Fig. 1). It was also found that cross-linking of the multilayers did not alter the surface homogeneity. ${ }^{37}$ Previous studies on mechanical properties of these crosslinked multilayers showed that the $E$-modulus of the native PLL/HA multilayers was $6.6 \mathrm{kPa}$, while cross-linking with different EDC concentrations increased the stiffness to 20.9 kPa for highly cross-linked layers. ${ }^{37}$

Quantitative analysis of AFM and SEM images revealed changes in gold area fraction and surface height profiles after multilayer deposition (Fig. 2). Before multilayer formation, the area fraction of gold structures increased from $17 \%$ (L) over $24 \%(\mathrm{M})$ to $28 \%(\mathrm{~S})$ with increasing angle of incidence. After deposition of PLL and HA, the area fraction of the nanofeatures significantly increased on all surfaces, ranging from $20 \%$ (L) over $26 \%(\mathrm{M})$ to $31 \%(\mathrm{~S})$, respectively. In addition, an obvious change in the progression of the height profiles was found (Fig. 2B). The height of the largest features significantly increased, while it significantly dropped to a lower value on medium and small features. Thereby, the decrease was largest on the smallest nanostructures.

\section{Surface wettability}

Water contact angle (WCA) measurements were used to determine the change in wettability after the different surface modification steps. Fig. 3A depicts the results of static WCA measurements, which reveals the hydrophilic nature of F-PEM. ${ }^{37}$ Cross-linking of multilayers resulted in an increased WCA in dependence on the EDC concentration. Thereby, F-PEM cross-linked with the highest EDC concentration (50 $\mathrm{mg} \mathrm{mL}^{-1}$ ) were significantly more hydrophobic than native PEM. On the other hand, pristine nanostructures exhibited a more hydrophobic character $\left(\mathrm{WCA}>65^{\circ}\right)$ with increasing WCA related to decreasing structure dimensions, which vanished after modification with SAMs obtaining a WCA in the range of $50^{\circ} \cdot{ }^{24}$ After modification with PEM, a further decrease in WCA was observed (Fig. 3B). However, it is interesting to note that in contrast to the F-PEM, WCA increased here with increasing cross-linking degree of PEM. A significant drop in WCA was observed on highly cross-linked samples in dependence on the structure dimensions. Thereby, nanostructures modified with PEM and cross-linked with the highest EDC concentration exhibited the lowest WCA. In addition, a significantly lower WCA was found on the highest cross-linked PEM on the smallest structures. 

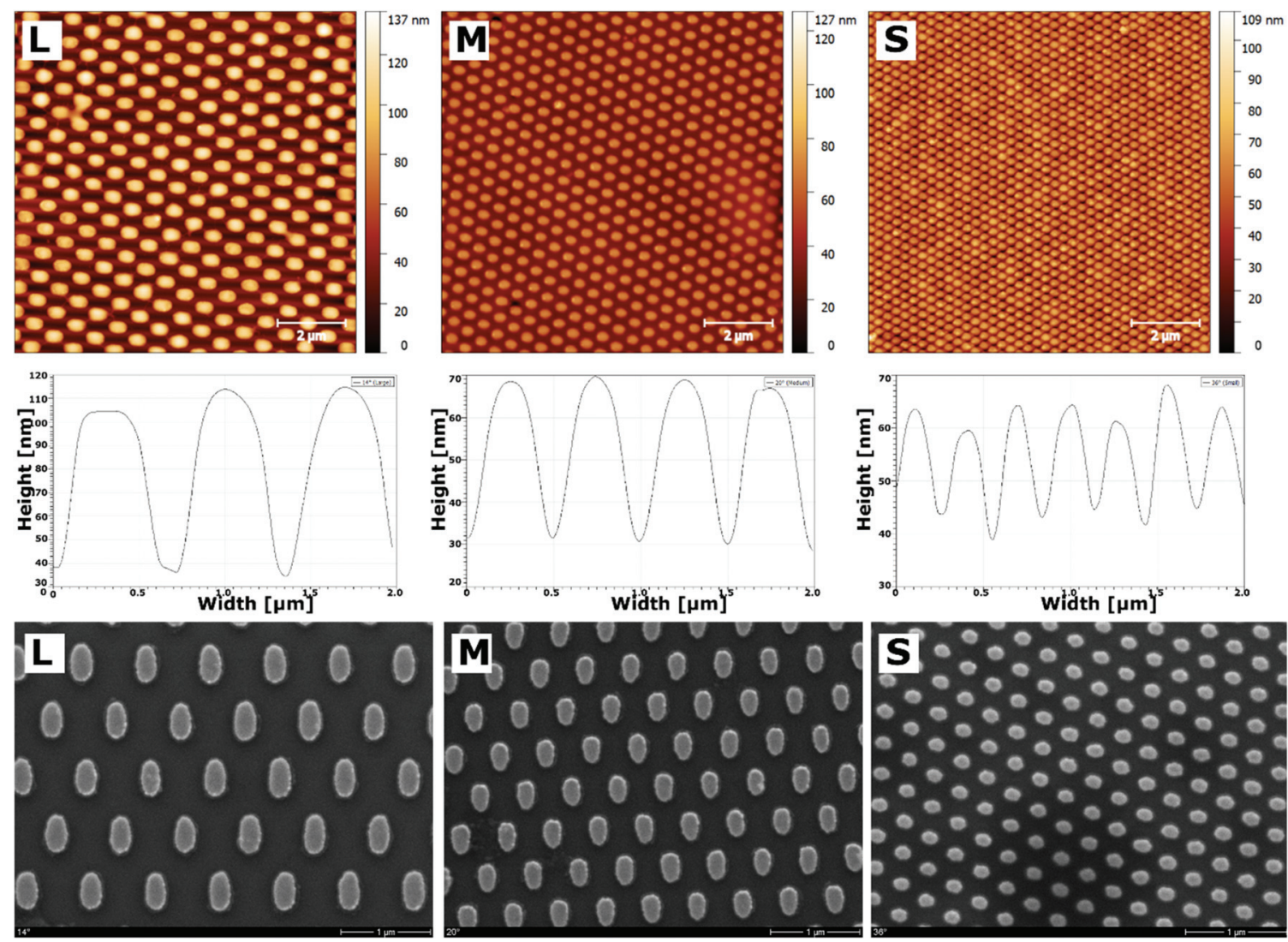
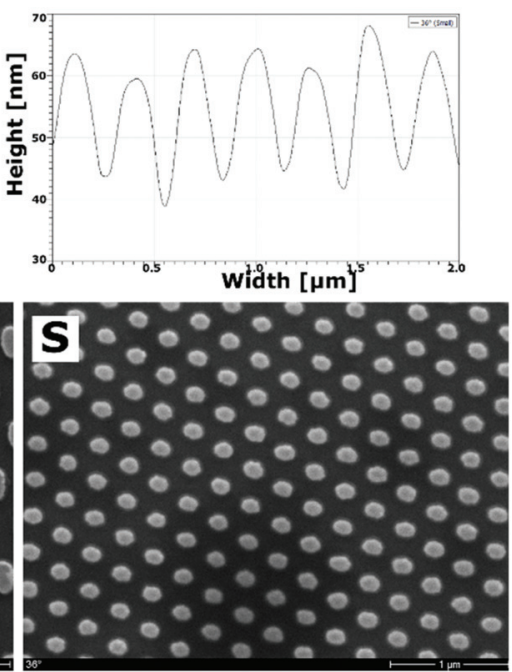

Fig. 1 AFM (top) and SEM (bottom) analysis of hexagonally arranged nanostructures. The structures of different dimension were obtained using three-beam laser interference lithography (LIL) at different angles of incidence (large $(\mathrm{L})=14^{\circ}$, medium $(\mathrm{M})=20^{\circ}$ and small $(\mathrm{S})=36^{\circ}$ ). Further, they have been modified with polyethylene glycol (PEG) and, subsequently, with mercaptoundecanoic acid (MUDA) and an additional multilayer system consisting of 11 bilayers of (PLL/HA) sandwiched by a primary PEI and terminal HA layer (PEI-[HA-PLL $\left.]_{11}-\mathrm{HA}\right)$. The height profiles obtained from AFM images (middle) show the progression of structures in lateral dimension [scale: $2 \mu \mathrm{m}$ ].

Results of dynamic WCA measurements were closely related to static WCA (Fig. S2 $\dagger$ ). Here it was seen that advancing WCA of native and $2 \mathrm{mg}$ EDC cross-linked PEM were significantly higher on large (L) structures compared to $\mathrm{M}$ and S. Nanostructures modified with PEM at the highest crosslinking degree exhibited the lowest advancing contact angles. The receding contact angles were at a similar level at all conditions. Hence, the difference in WCA hysteresis was mainly attributed to the difference in advancing contact angles. Here, the largest differences were found on native as well as low cross-linked PEM. The hysteresis was always lowest for the intermediate structures, except for the highest cross-linking degree. However, significant differences in dependence on the feature dimensions could not be revealed.

\section{Cell adhesion studies}

Adhesion and spreading of human adipose-derived stem cells (hADSC) on F-PEM and S-PEM with various cross-linking degree $\left(2,10\right.$, or $\left.50 \mathrm{mg} \mathrm{mL}{ }^{-1} \mathrm{EDC}\right)$ were investigated after $4 \mathrm{~h}$ and $24 \mathrm{~h}$ of incubation. There, vinculin in focal adhesions (FA), actin cytoskeleton, and the nucleus were stained immunocytochemically and image analysis was performed to determine count, area, and aspect ratio of hADSC. Additionally, focal adhesion kinase (FAK) as well as the small GTPase RhoA were stained immunocytochemically to determine signal transduction processes under short-term culture conditions in a qualitative and semi-quantitative manner.

CLSM images revealed differences in the expression of FA plaques and actin formation in dependence on the surface composition and culture time. On F-PEM, cell spreading increased with cross-linking degree after $4 \mathrm{~h}$ (Fig. S3†). However, vinculin organization in FA was absent in cells seeded on native and little cross-linked PEM ( $2 \mathrm{mg}$ EDC). In addition, actin was not fibrillized and dispersed in the cytoplasm, even after $24 \mathrm{~h}$ incubation (Fig. 4). Cells seeded on PEM with the highest cross-linking degree showed a slightly enhanced spreading with beginning actin polymerization. Still, actin expression was weak after $24 \mathrm{~h}$. In addition, the expression of vinculin in FA was not observed in cells on native and low cross-linked PEM after both incubation times. Cells seeded on highly cross-linked PEM showed signs of vinculin expression, which was enhanced after $24 \mathrm{~h}$ incubation. The quality of adhesion differed to a higher extent in hADSC cultured on S-PEM particularly in dependence on the cross- 
A

linking degree, but also partly on the structure dimensions. After $4 \mathrm{~h}$, actin polymerization as well as vinculin expression was low and independent of cross-linking degree and nanostructure size (Fig. S3†). However, actin fibers were circumferentially organized on native S-PEM with larger feature dimensions $\left(14^{\circ}, \mathrm{L}\right.$ and $\left.20^{\circ}, \mathrm{M}\right)$. Additionally, vinculin in FA was rarely expressed. In contrast, cells seeded on non-cross-linked PEM on nanostructured samples with the smallest feature dimension $\left(36^{\circ}, S\right)$ exhibited longitudinal actin fibers and expressed vinculin in the cell periphery. Besides the timedependent increase in cell area, cells seeded on native and low cross-linked PEM on nanostructures were more elongated than cells on the highly cross-linked samples after $24 \mathrm{~h}$ incubation (Fig. 4). Additionally, actin bundles were organized longitudinally and vinculin in FA was found in the periphery of the cells. However, a clear trend of cell adhesion quality in dependence on the nanostructure dimension was not observed. Nevertheless, cells seeded on highly cross-linked nanostructured samples had different morphology. Actin was more condensed in the cell periphery, especially on the smallest fea-
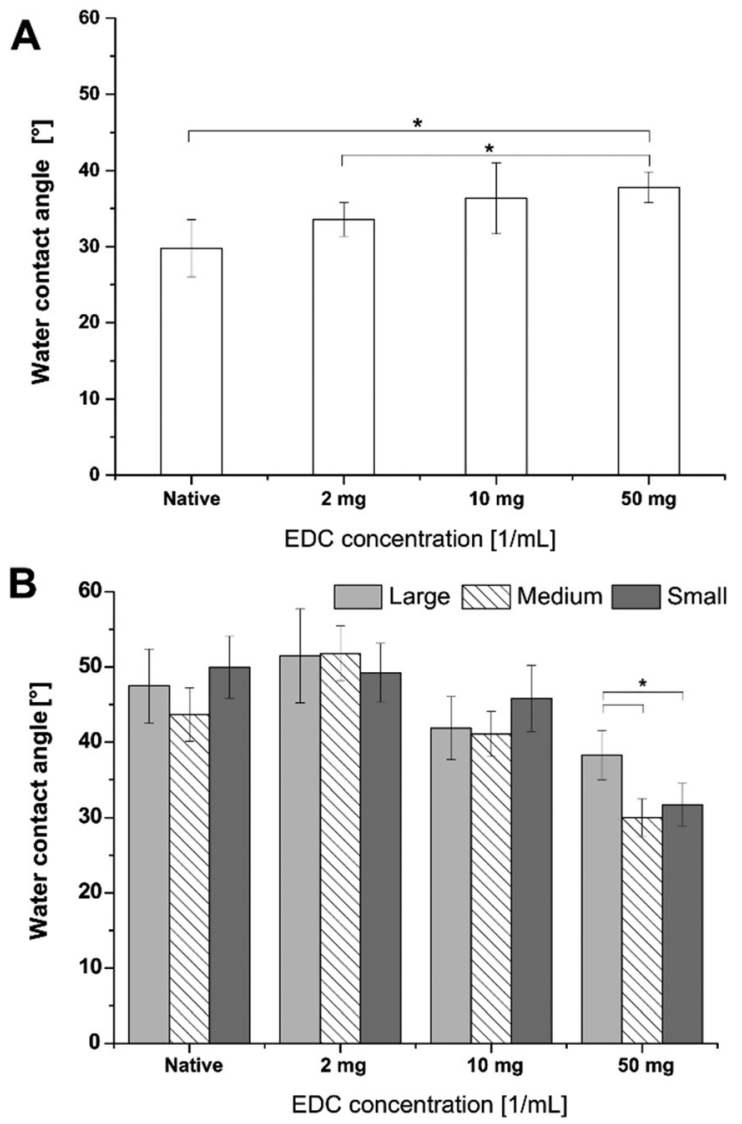

Fig. 3 Results of static water contact angle (WCA) analysis. The wettability of F-PEM (A) as well as S-PEM (B) was determined using static WCA measurements. Further, the multilayer system was cross-linked with different EDC concentrations $\left(2,10\right.$, or $\left.50 \mathrm{mg} \mathrm{mL}^{-1}\right)$ or used native $[p<0.05]$.

tures. It seemed that filopodia formation was increased on native and low cross-linked PEM on nanofeatures, while lamellipodia formation was enhanced on highly cross-linked PEM on the smallest features. Interestingly, the determination of the length of FA showed clear trends. It was found that FA were decreasing in cells on all feature dimensions with increasing cross-linking degree (Fig. 5A). In addition, it seemed that FA length also followed the feature size, meaning that FA were smaller on the smallest features, which was seen on all crosslinking degrees.

The quantification of cell area and aspect ratio were taken as indicators for cell spreading on the different surfaces. After $4 \mathrm{~h}$ incubation, cell spreading significantly increased with increasing cross-linking degree on F-PEM (Fig. S4A †े). Moreover, spreading of hADSC highly increased on PEM crosslinked with the highest EDC concentration after $24 \mathrm{~h}$, while on native PEM and layers cross-linked with $2 \mathrm{mg} \mathrm{mL}^{-1}$ EDC no change was observed (Fig. 6A). Overall, the enhanced cell spreading with increased EDC concentration indicated a direct relation between cross-linking degree and cell spreading. This trend was also found during determination of the aspect ratio, especially after $24 \mathrm{~h}$ (Fig. 6B). Here, the aspect ratio increased 

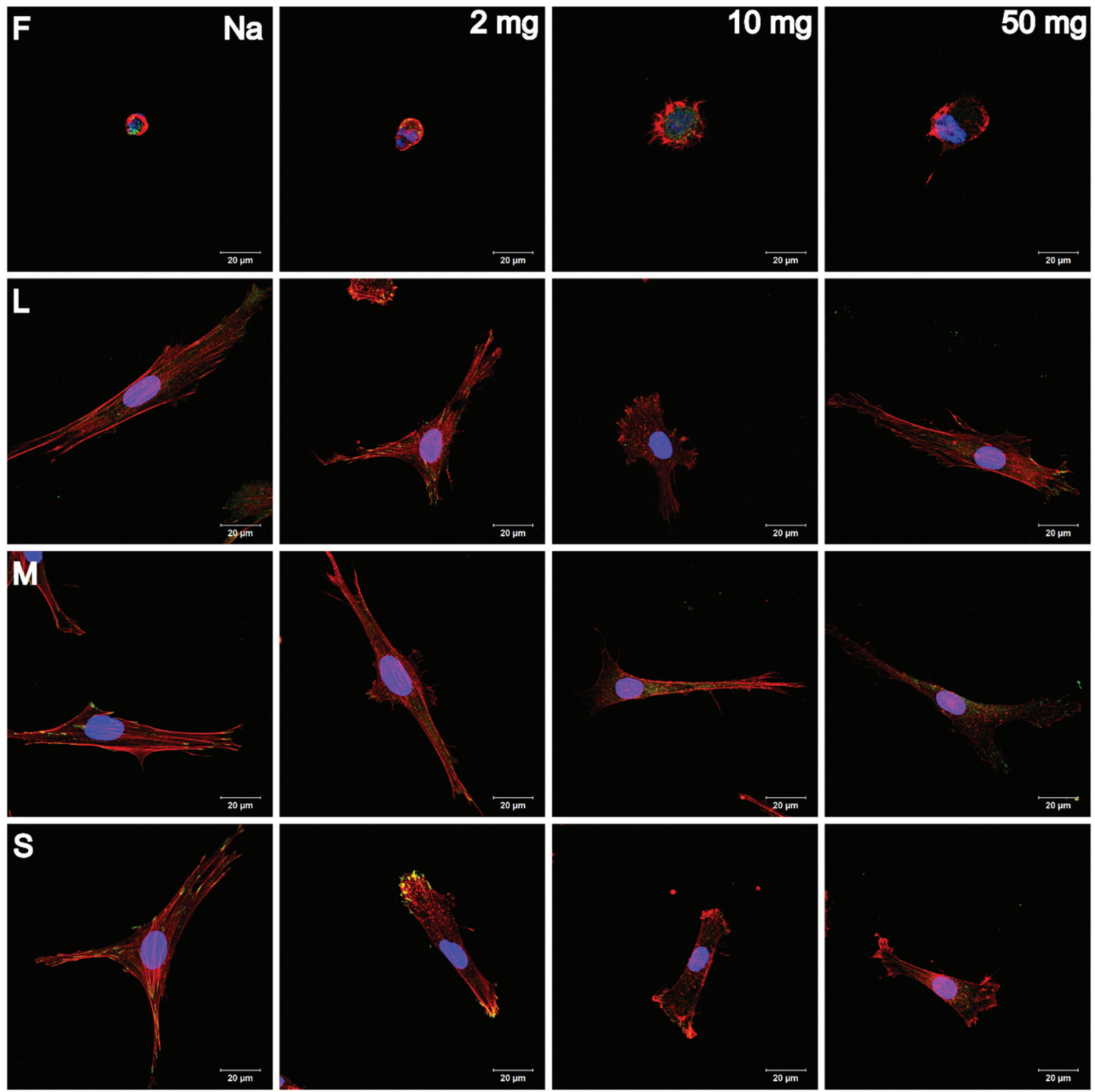

Fig. 4 CLSM images of adherent hADSC cultured on flat (F) multilayers or on S-PEM. The multilayers were cross-linked with different EDC concentrations $\left(2,10\right.$, or $50 \mathrm{mg} \mathrm{mL}^{-1}$ ) or used native (Na). Afterwards, the cells were cultivated for $24 \mathrm{~h}$ and stained for the nucleus (blue), actin cytoskeleton (red), and vinculin in focal adhesions (green). The letters L, M, and S denote the feature dimensions large, medium, and small [scale: $20 \mu \mathrm{m}$ ].

with increasing EDC concentration, which was still not observed after $4 \mathrm{~h}$ (Fig. S4B $\dagger$ ). However, cells seeded on native as well as on PEM cross-linked with the lowest EDC concentration did not show a change in aspect ratio in relation to the incubation time. In contrast, cells seeded on highly crosslinked PEM were highly spread after $24 \mathrm{~h}$.

Stem cells seeded on S-PEM showed an inverse behavior. After $4 \mathrm{~h}$ incubation, no trend in cell area was found, neither in dependence on the cross-linking degree nor the structure size (Fig. S4A $\dagger$ ). Only cells on native PEM spread to a higher extent. However, a slight trend in the aspect ratio was found in dependence on the cross-linking degree, which means that cells were more elongated on native PEM and more round on highly cross-linked PEM (Fig. S4B $\dagger$ ). After prolonged incubation time, a clear trend in cell area as well as aspect ratio was found, even though the variation of calculated values was high (Fig. 6). When looking at the mean and median values, a decreasing trend was found with increasing cross-linker concentration, which means that cells spread more on less crosslinked S-PEM. However, the range of the cell area was sometimes large leading to insignificant results. Still, the observed trend found during quantification of the aspect ratio after $4 \mathrm{~h}$, was also found after $24 \mathrm{~h}$, but with a stronger progression (Fig. 6B). Again, the large range of the single results prevented statistically significant values. Here, cells on native and low cross-linked samples showed enhanced elongation, but independent of the nanostructure dimensions.

Signal transduction processes were investigated by immunocytochemical staining of the small GTPase RhoA and the focal adhesion kinase (FAK) after $4 \mathrm{~h}$ (Fig. S5 $\dagger$ ) and $24 \mathrm{~h}$ 
A

B
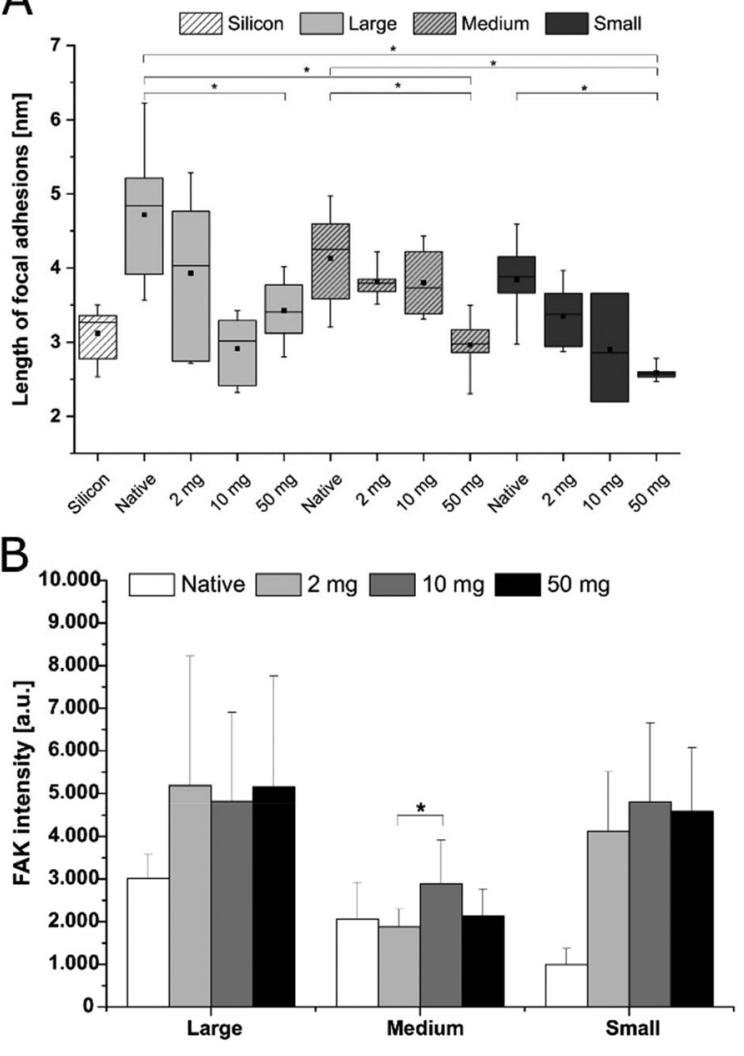

Structure dimension

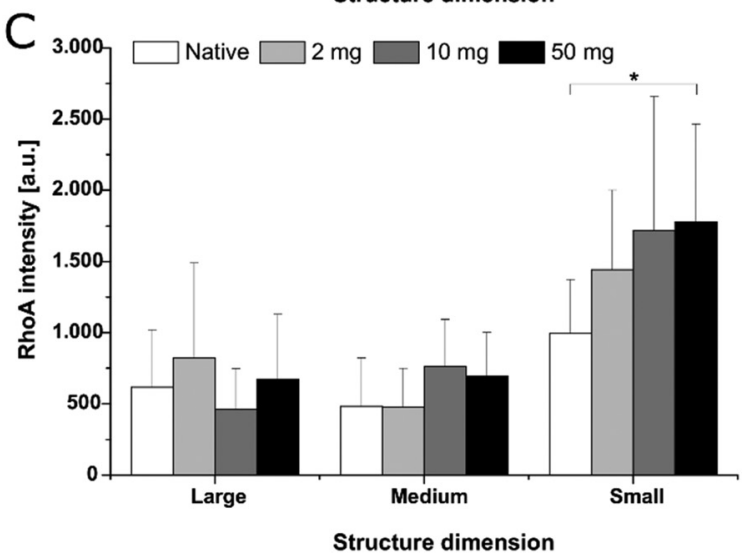

Fig. 5 Quantification of focal adhesion length (A), RhoA (B) and focal adhesion kinase (FAK) (C) of hADSC cultured on S-PEM. The multilayer system was cross-linked with different EDC concentrations $(2,10$, or $50 \mathrm{mg} \mathrm{mL}^{-1}$ ) or used native. Thereafter, the cells were incubated for $4 \mathrm{~h}$ $(\mathrm{B}, \mathrm{C})$ and $24 \mathrm{~h}(\mathrm{~A})$ on the different surfaces (large $-14^{\circ}$, medium $-20^{\circ}$ and small $-36^{\circ}$ ) and image analysis was performed $[p<0.05]$.

(Fig. 7) of incubation. The expression of both markers enhanced in compliance with the EDC concentration as well as the incubation time on F-PEM. Cells seeded on native and low cross-linked PEM films were not able to form FA plaques. In contrast, hADSC seeded on highly cross-linked PEM showed enhanced presence of FAK. These FA plaques were located at the cell periphery and increased with prolonged incubation time. Quantitative evaluation of intensity of FAK
A
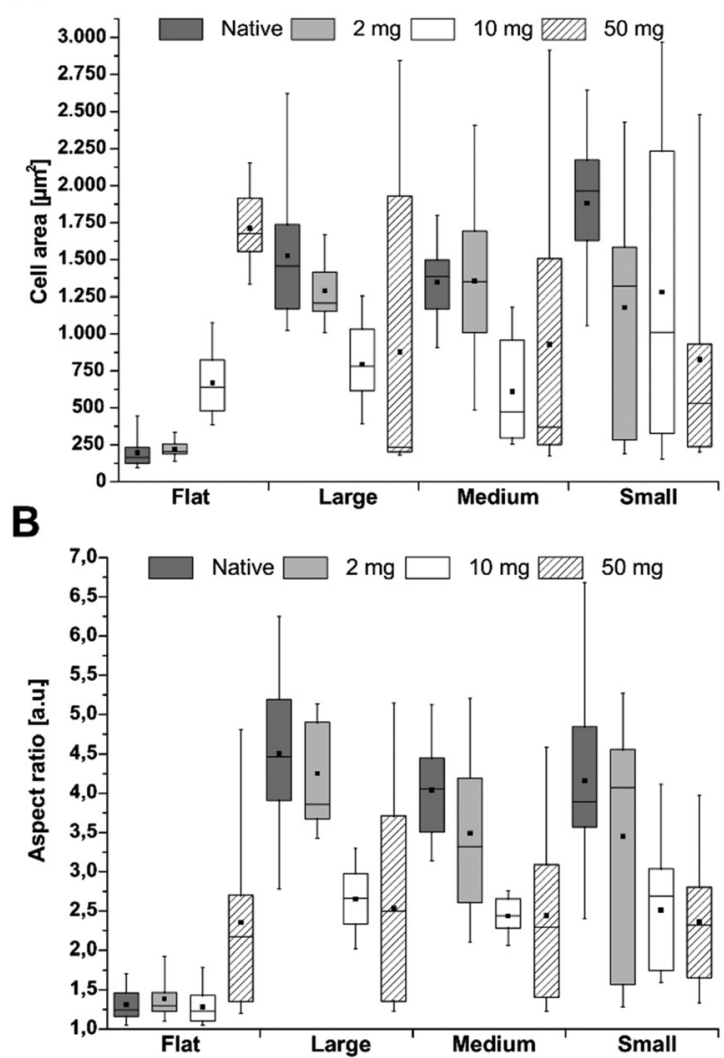

Fig. 6 Quantification of (A) cell area and (B) aspect ratio of hADSC cultured on F-PEM or S-PEM (large $-14^{\circ}$, medium $-20^{\circ}$ and small $-36^{\circ}$ ). The multilayers were cross-linked with different EDC concentrations (2, 10 , or $50 \mathrm{mg} \mathrm{mL}^{-1}$ ) or used native. Images were taken after $24 \mathrm{~h}$ incubation and analysis was performed using ImageJ software with investigation of at least 10 images per condition. The box indicates the $25^{\text {th }}$ and $75^{\text {th }}$ percentile, the median (dash) and mean value (black square), respectively, whereas the $95-5 \%$ confidence interval is represented by the whiskers.

expression in cells after $4 \mathrm{~h}$ adhesion revealed no significant differences between structure dimensions (S, M, L), but a significantly lower expression on the native S-PEM compared to those cross-linked with concentration of EDC (10 and 50

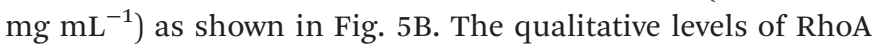
were also enhanced with longer cultivation time and a random dispersion of RhoA molecules was noticed on highly cross-linked samples. Quantitative analysis of RhoA in cells revealed higher intensities in cells seeded on smaller feature dimensions (S), but also an increased activity of RhoA in hADSC that were seeded on higher cross-linked samples when compared with those seeded on native S-PEM (see Fig. 5C). In general, the expression of FAK and RhoA molecules was more prominent in cells seeded on the smallest feature dimensions, especially after $24 \mathrm{~h}$. Overall, native and low cross-linked PEM on small structures $\left(36^{\circ}\right)$ resulted in well-expressed FAK and RhoA molecules localized in the cytoplasm (Fig. 7). 

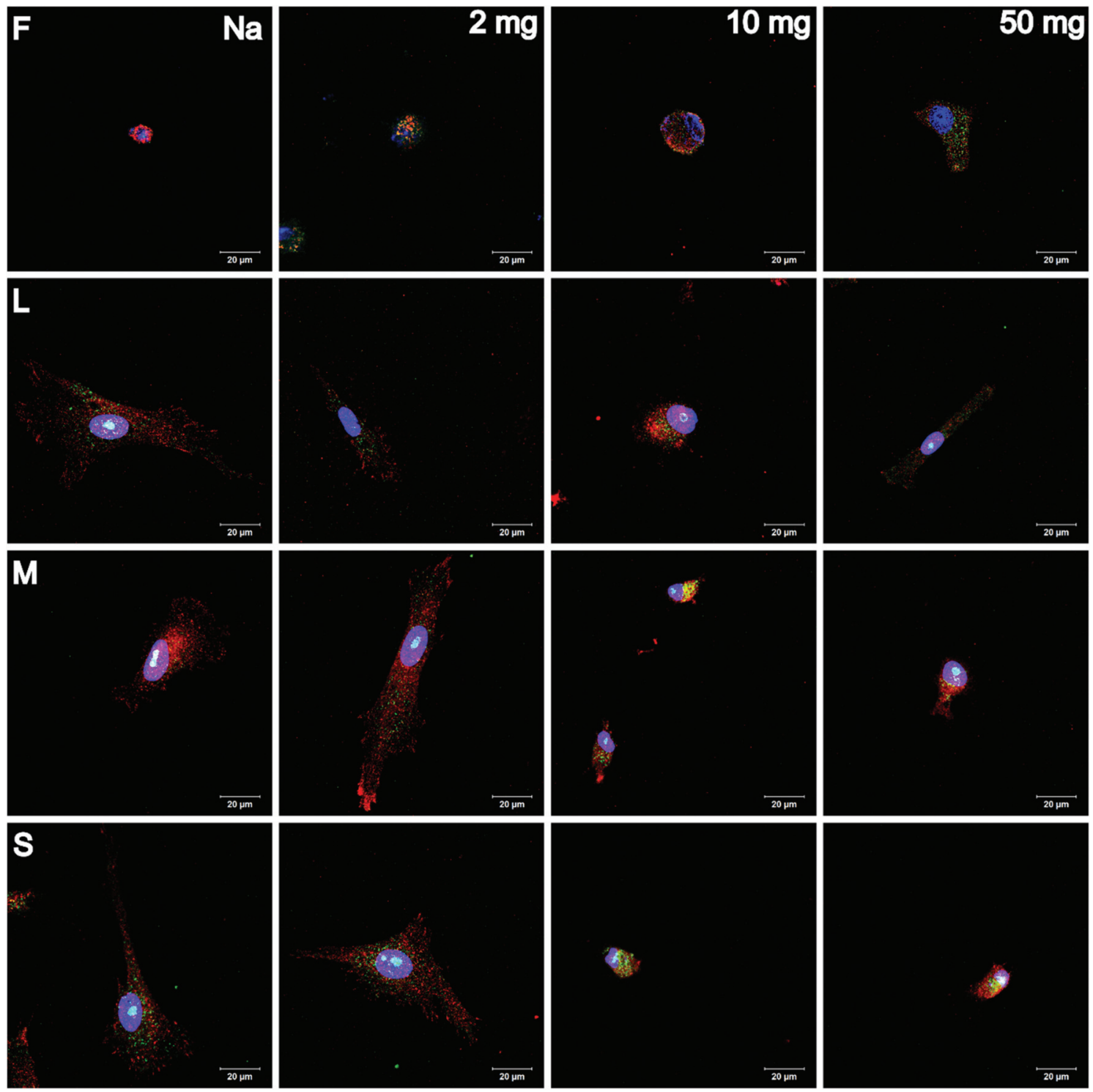

Fig. 7 CLSM images of adherent hADSC cultured on flat (F) multilayers or on S-PEM. The multilayers were cross-linked with different EDC concentrations $\left(2,10\right.$, or $\left.50 \mathrm{mg} \mathrm{mL}^{-1}\right)$ or used native $(\mathrm{Na})$. Afterwards, the cells were cultivated for $24 \mathrm{~h}$ and stained for the nucleus (blue), focal adhesion kinase (FAK, red), and the small GTPase RhoA (green). The letters L, M, and S denote the feature dimensions large, medium, and small, respectively [scale: $20 \mu \mathrm{m}$ ].

\section{Cell differentiation studies}

Human ADSC differentiation on flat PEM and nanostructures modified with native and highly cross-linked PEM was induced with specific differentiation media after proliferation of cells in control medium for seven days. After 21 days of induction, hADSC were immunocytochemically stained for specific differentiation markers.

Adipogenic differentiation was investigated by visualization of perilipin and glucose transporter 4 (GLUT4). It was found that cells did not grow to confluency after 28 days of culture (Fig. S6†). Still, single, but spread spindle-shaped cells were observed on S-PEM, while cells were still round on F-PEM at both cross-linking degrees. Moreover, adipogenic differen- tiation could not be proven on F-PEM, since perilipin staining was absent. Still, GLUT4 was visible across the cytoplasm. However, it was expressed to a higher extent in cells cultured on S-PEM. Further, perilipin was distributed weakly across the whole cytoplasm, whereas GLUT4 was found to accumulate at the periphery of the cells. Cells cultured on native films showed an enhanced spreading independent of the structure dimension if compared to the cross-linked PEM. Further, GLUT4 expression seemed to be enhanced on highly crosslinked PEM, but independent of the feature dimension. Overall, the typical formation of fat vacuoles was absent in cells on all surfaces.

Chondrogenic differentiation was investigated by visualization of aggrecan and collagen II (Fig. 8). First, no cells and 

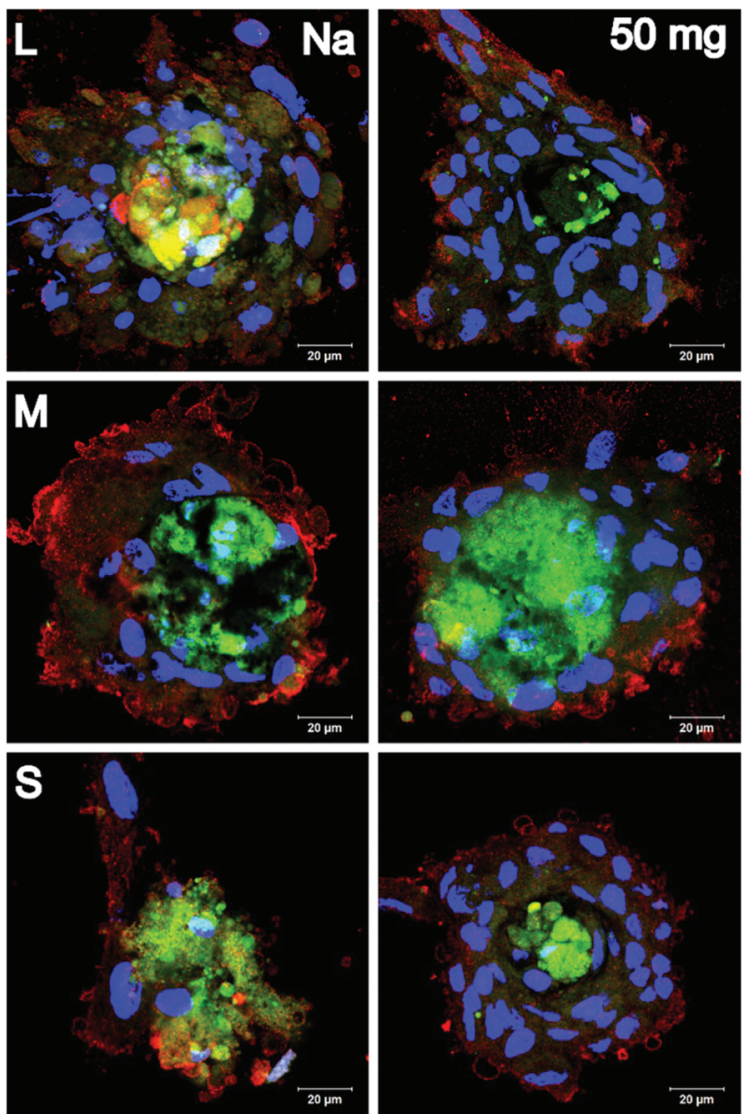

Fig. 8 Visualization of chondrogenic differentiation of hADSC Nanostructured substrates were modified with (PLL/HA) multilayers and these layers were cross-linked with $50 \mathrm{mg} \mathrm{mL}^{-1} \mathrm{EDC}$ or used native $(\mathrm{Na})$. After 21 days of induction, hADSC were stained for specific differentiation markers. Here, aggrecan (green) and collagen II (Col II, red) were stained together with the nucleus (blue). The letters $L, M$, and $S$ denote the feature dimensions large, medium, and small, respectively [scale: $20 \mu \mathrm{m}]$.

hence no chondrogenic differentiation were observed on F-PEM. However, both markers were visible in cells cultured on S-PEM. Interestingly, the cells formed clusters rather than growing in monolayers. These clusters were largest on the largest features and were reduced in size with decreasing feature dimension. In addition, the PEM cross-linking degree seemed to have no obvious influence on the cluster size. The investigation of specific differentiation markers revealed that aggrecan was uniformly distributed within the cell clusters, while collagen II was primarily accumulated at the periphery, but also found within the cluster. The more cells integrated into clusters, the higher was the collagen II secretion, meaning that the lowest amount was found on the smallest nanostructures.

Osteogenic differentiation was investigated by visualization of collagen I and osteocalcin (Fig. 9). It was found that cells did not grow to confluency on F-PEM, no matter if cross-linked or not. Moreover, osteogenic differentiation was not observed. In contrast, cells on S-PEM preferentially grew in monolayers,
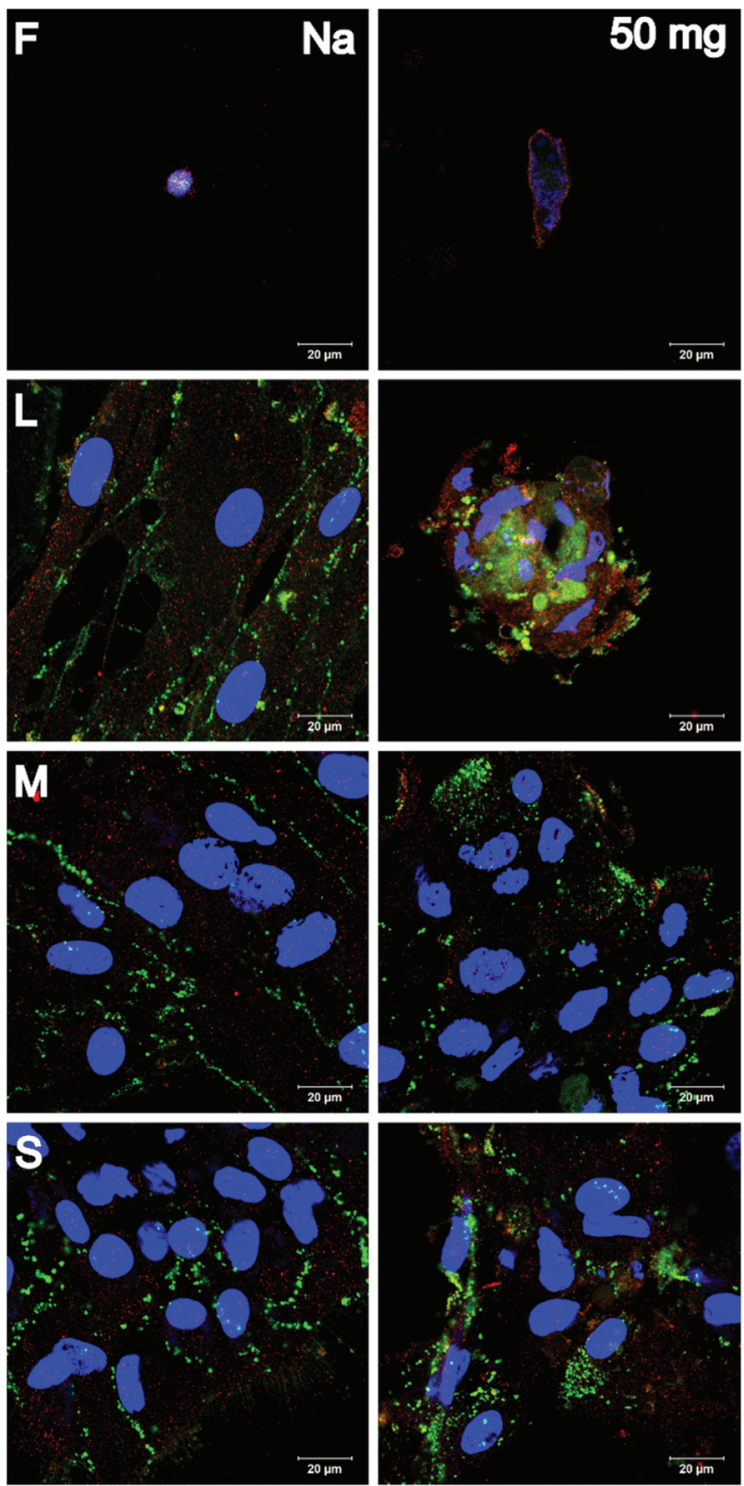

Fig. 9 Visualization of osteogenic differentiation of hADSC. Nanostructured substrates were modified with (PLL/HA) multilayers and these layers were cross-linked with $50 \mathrm{mg} \mathrm{mL}^{-1} \mathrm{EDC}$ or used native $(\mathrm{Na})$. After 21 days of induction, hADSC were stained for specific differentiation markers. Here, collagen I (Col I, green) and osteocalcin (red) were stained together with the nucleus (blue). The letters $L, M$, and $S$ denote the feature dimensions large, medium, and small, respectively [scale: $20 \mu \mathrm{m}]$.

especially on native PEM. The cross-linked PEM caused the cells to form clusters with larger ones on the smallest features. Further, it seemed that also on the native PEM cells affiliated close to each other to form pre-clusters indicated by the accumulation of nuclei within the monolayer. Differences were found additionally in the expression of osteogenic markers. Osteocalcin was accumulated in spots across the cell body, independent of cells in monolayers or clusters. Further, collagen I was aggregated predominantly in the cell periphery in cell monolayers, preferentially at areas of cell-cell-contact. In 
cell clusters, it was distributed evenly across the whole cell body.

\section{Discussion}

In this study, three-beam LIL was used to design hexagonally arranged nanostructures of gold, which represents a relatively simple and cost-efficient technique. ${ }^{24}$ Briefly, large areas of well-defined and consistent patterns can be produced in the nanoscale in a relatively short time, which makes LIL an attractive technique. ${ }^{39}$ Regular patterns can be produced whose sizes dependent on the wavelength of the used light from a monochromatic laser source and the angle of incidence. We have shown recently that size and periodicity of ordered nanostructures are perfectly in line with theoretical predictions, which underlines the precision of LIL. ${ }^{24}$ Another advantage of the approach demonstrated here is the combination of two different materials, silicon or any other reflective materials with gold vapor deposition after development of the photoresist. This allows a binary modification of surfaces as it was done by passivation of the silicon base material with an organo-silane with long-chain PEG and activation of gold nanostructure by a thiol with a terminal carboxylic group (MUDA), which provides also a negative charge useful for adsorption of molecules and a chemical group for further covalent coupling reactions. Hence, these steps allow the selective modification of gold structures with proteins or other molecules, while the surrounding surface possesses protein and cell-repulsive properties. $^{24}$ Here we introduced an additional feature to the system, adding a viscoelastic element to the nanostructures by formation of a multilayer system composed of PLL and HA using a simple and versatile spraycoating process. ${ }^{40}$ Subsequent chemical cross-linking of multilayers was able to change viscoelasticity to have either a soft or stiffer multilayer coating. ${ }^{37}$

SEM and AFM investigations showed that nanostructures were still visible after multilayer assembly. Image analysis revealed that the surface coverage with nanostructures not only increased with increasing angle of incidence from large (L) to small (S) feature dimensions, but also after multilayer assembly on them. This indicates that the size of structures increased with the accumulation of PEL, which is probably due to adsorption of PEL on the side of the gold nanostructures that have a height of about $60 \mathrm{~nm}$. In our previous studies, we could show that proteins and PEL preferentially adsorbed on the nanostructures and did not accumulate on the passivated areas to a high extent. ${ }^{24,41}$ Further, here it was found that the height of nanostructures increased about $30 \mathrm{~nm}$ for the largest feature dimensions (L), while it decreased about $30 \mathrm{~nm}$ for $\mathrm{M}$ and $\mathrm{S}$ structures measured on dry samples. This indicates that multilayers did not assemble exclusively on the mean size (M) and small (S) nanostructures, but also started to cover the surrounding passivated area, which might be due to the growing of multilayers from the edges of nanostructures. Island-like growth of multilayer films in the nanoscale is one of the ideas of multilayer film formation and supports this assumption. ${ }^{42}$ Furthermore, the height of nanostructures was increased on large features $(\mathrm{L})$, which indicates that a fusion of multilayers on the surrounding substratum does not occur at larger distances, which supports also the claim that passivation by PEG is an effective measure to prevent multilayer formation. Still, the nanostructures were detectable for all feature dimensions as seen by AFM profiles in Fig. 1, in a dry state. If we consider that these kind of PLL/HA multilayers have a considerably higher thickness in a hydrated and swollen state of about 200-300 $\mathrm{nm}$ as measured in our previous investigation, ${ }^{37}$ we can assume that adhesion of cells will occur predominantly with multilayers on top of nanostructures and not with the surrounding silicon area due to the inability of the cell membrane to bridge height differences larger than $70 \mathrm{~nm}$ by bending. ${ }^{43}$

It is interesting to note that F-PEM became less hydrophilic with increasing cross-linker concentration, which was attributed previously to the formation of amide bonds between carboxylic groups of HA and primary amino groups of PLL. ${ }^{44}$ By contrast, the cross-linking of PLL/HA multilayers on nanostructures rendered the surfaces more hydrophilic independent of the nanostructure size. The higher WCA of structured vs. flat PEM was particularly obvious for the native and low cross-linked $\left(2 \mathrm{mg} \mathrm{mL}^{-1}\right)$ multilayers. Particularly advancing WCA (see Fig. S2 $\dagger$ ) were larger for larger structure dimensions (L) compared to $\mathrm{M}$ and $\mathrm{S}$. This indicates that entrapment of air could be responsible for the higher WCA of nanostructured PEM vs. flat according to the Cassie-Baxter model. ${ }^{45}$ Increasing the cross-linker concentration on nanostructured surfaces led to a decrease of WCA opposing the rise of WCA on flat PEM. Results of dynamic WCA measurements showed a decrease of contact angle hysteresis for the highest cross-linker concentration $\left(50 \mathrm{mg} \mathrm{mL}^{-1}\right)$, which indicates a more homogenous surface leading possibly to lesser air entrapment due to a structuring effect of the surface corresponding to the Wenzel approach. Hence, this in turn means that rougher, hydrophilic surfaces have lower WCA than smooth. ${ }^{46}$

\section{Cell adhesion studies}

Strength and extent of adhesion and spreading of cells can strongly influence their growth and differentiation..$^{30,47-49}$ Spreading of mesenchymal stem cells was shown to promote osteogenic differentiation, while little spread cells underwent adipogenic differentiation. ${ }^{47}$ In contrast to our previous studies, ${ }^{22,24}$ we show here that spreading, growth, and differentiation of cells was influenced to a lesser extent by size and periodicity of nanostructures, but to a higher extent by the modification with multilayers and the cross-linker concentration. We confirm also results of a previous study showing increased, but limited spreading of hADSC with higher crosslinking degree on F-PEM, attributed to the increased $E$-modulus, which is in line with other cross-linked multilayer systems. ${ }^{30,37,38,50}$ The cells had a rather low aspect ratio, indicating that the F-PEM surfaces appeared homogeneous to the cells, leading to an equal spreading in all directions, which 
means low polarization. Cell area and aspect ratio were much higher when cells were plated on native or little cross-linked multilayers ( $2 \mathrm{mg} \mathrm{mL}^{-1}$ ) prepared on nanostructures (S-PEM), which is strikingly different from their behavior on F-PEM. The better spreading goes along with the higher water contact angles $\left(\theta \sim 50^{\circ}\right)$ i.e. a lower wettability, which generally promotes adsorption of medium-derived or cell-secreted proteins and hence cell adhesion. ${ }^{51} \mathrm{~A}$ further difference between F-PEM and S-PEM is the presence of a structured surface. The F-PEM may resist lesser versus contractile forces that adhering cells generate upon attachment due to the homogenous nature of the rather thick swollen film, which leads to stronger deformation of films comparable to studies from Engler et al. and Discher et al. on hydrogels. ${ }^{52,53}$ Hence, cells probing the underlying substratum would fail to develop sufficient tension to assemble FA and cytoskeletal components (Fig. 10). ${ }^{53}$ By contrast, S-PEM appear as discrete elements that cannot be translocated like material in the homogenous, flat films. Hence, more tension can be developed by cells, which supports to the formation of FA and assembly of cytoskeletal elements and stronger polarization of cells. Accordingly, FA

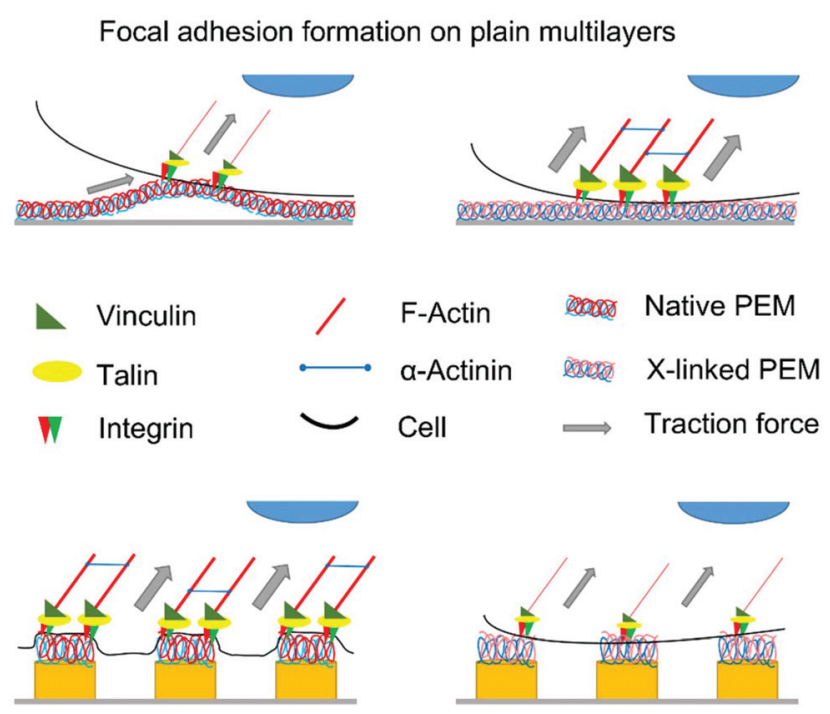

Focal adhesion formation on PEM-modified nanostructures

Fig. 10 Schematic representation of the influence of surface parameters on focal adhesion formation and actin polymerization. On flat, non-cross-linked multilayers (PEM, top left), low contractile forces can be established due to the soft, pliable nature of the native multilayers. Hence, the cells can contract the multilayer, which impairs stable focal adhesion formation. In contrast, on highly cross-linked, flat PEM (top right), high contractile forces are generated due to the lower elasticity, resulting in stable focal adhesions and strong actin polymerization. On nanostructures modified with non-cross-linked PEM (bottom left), the cells sense discrete multilayers attached to the nanostructures, which cannot be pulled away, leading to stable adhesion plaques and strong actin fibers. In contrast, the higher wettability of highly cross-linked PEM on nanostructures (bottom right) together with the negative charge results in lower protein adsorption and, hence, lesser ligation of integrins leading to weaker expression of focal adhesions and formation of actin stress fibers. formation and actin polymerization was superior compared to a flat, homogenous PEM film (Fig. 10). This was also related to presence of FAK in FA and stronger expression of RhoA. FAK is a tyrosine kinase located in FA regulating adhesion, shape, migration, and differentiation of cells. ${ }^{54-56}$ RhoA is a small GTPase that couples with ROCK, a downstream regulator of myosin-light chain phosphorylation leading to contractile forces. ${ }^{57,58}$ The sensing of elasticity is essential for mechanotransduction and, hence, influenced by activation of RhoA/ ROCK that results in development of cytoskeletal tension. ${ }^{58}$ It was also observed that increasing the cross-linking of the S-PEM led to higher hydrophilicity and, hence, more water binding on the film surfaces, which might counteract the adhesive properties of the system due to a lower propensity of protein adsorption and, hence, lesser cell spreading and FA formation. ${ }^{51}$ The shorter length of FA on highly cross-linked S-PEM supports this assumption. Further, the reduced FA length with decreasing feature dimension is also reasonable due to less space for the formation of stable anchorage points. $^{22}$ Another aspect of the S-PEM film is related to the charge density of PEL because a recent study comparing the presence of charged groups on flat and nanostructured surfaces found an amplifying effect of nanostructures on cell adhesion and spreading. ${ }^{59}$ Overall, it was well visible that the cells could still sense the topography underneath the PLL/HA multilayers even though their thickness of about $200-300 \mathrm{~nm}$ was considerably higher than the nanostructures. ${ }^{37}$

\section{Cell differentiation}

Studies on cell differentiation by immunofluorescence staining of adipogenic, chondrogenic, and osteogenic markers were performed here in the presence of differentiation medium to see whether the topography and mechanical properties of substrata provide a microenvironment that either promotes or inhibits the lineage commitment of hADSC. Since FAK and RhoA represent important signal transducers for lineage commitment of stem cells, ${ }^{47,56}$ their organization and expression was interesting as well. Here, hADSC cultured on native S-PEM expressed FAK and RhoA proteins to a lower extent than cells on highly cross-linked layers, when analyzed in a quantitative manner. Since high levels of active RhoA are related to osteogenic differentiation, a promoting effect of topography, but also mechanical properties of the substratum can be assumed. ${ }^{47,56}$ By contrast, in our previous study where hADSC did not show signs of osteogenesis on pristine nanostructures, ${ }^{24}$ hADSC expressed collagen I and osteocalcin on S-PEM here, particularly on the highly cross-linked samples. The osteogenic differentiation was obviously related to the presence of a structured substratum since in a previous study on F-PEM, we did not see any lineage development in this direction due to the soft nature of the native PLL/HA PEM with an $E$-modulus of about $5 \mathrm{kPa} .{ }^{37}$ Here, the more hydrophilic, but stiffer cross-linked layers led to more condensation of cells and nodule formation, especially on the largest features, which was also related to higher expression of RhoA. These nodules are a typical sign of osteogenesis. ${ }^{60}$ Hence, the combi- 
nation of substrate stiffness and wetting properties seems to be a regulator of the differentiation in dependence on the culture conditions i.e. the presence of osteogenic medium supplements. On the other hand, in the presence of chondrogenic medium hADSC started to form cell clusters indicating chondrogenic differentiation with condensation of cells. ${ }^{61}$ Obviously, these clusters were largest on the largest features dimensions (L) on native and highly cross-linked samples. However, cross-linking resulted in smaller clusters, indicating that cells on stiffer, more wettable surfaces have a lower propensity for chondrogenic differentiation. ${ }^{50}$ Still, collagen II and aggrecan were expressed to similar extent, which gives also evidence for the lineage commitment. In such aggregates, $\mathrm{N}$-cadherin is upregulated to form cell-cell contacts with subsequent intracellular signaling to start the transition from progenitor cells to fully committed chondrocytes. ${ }^{62}$ Even though some expression of adipogenic markers was detected in hADSC on S-PEM, a complete differentiation could not be determined. High levers of RhoA in hADSC may counteract lineage commitment into adipogenic phenotype despite the presence of adipogenic inducers. The strong polarization of cells due to the structured and partly cross-linked PEM with a stiffer surface inhibited obviously adipogenesis, since adipocytes typically keep a round shape with decreased cytoskeletal assembly and fat vacuoles are formed, where perilipin is located. ${ }^{63}$ Such vacuoles were not found here. However, due to the softer nature of native PLL/HA films, marker expression was increased if compared to cross-linked PEM. It should be also noted that such analysis of cell differentiation was not possible on F-PEM due to the limited low adhesiveness and growth of cells, which did not permit immunofluorescence staining and investigation with CLSM. Indeed, we observed in our previous work adipogenic differentiation of hADSC on native F-PEM by histochemical staining. ${ }^{37}$

\section{Conclusion}

The aim of this study was to study the combination of nanostructures of different periodicities with a viscoelastic element based on native or cross-linked multilayers to study the effect of topography and mechanical properties on hADSC fate. Hexagonally arranged nanostructures of gold with different size and periodicity were fabricated using LIL, selecting different angles of incidence. In addition, a multilayer system composed of the biogenic molecules PLL and HA was assembled on the nanostructures using the spray-assisted LbL technique to control surface properties by subsequent crosslinking. The combination of both techniques resulted in exceptional systems with unique properties. While cells were spreading to a higher extent on F-PEM with increasing crosslinking degree, hADSC round up with increasing cross-linking degree on nanostructured surfaces. Obviously, the structures beneath not only affected cell adhesion, but also their differentiation. Hence, future scaffold design could use the obtained results as a support, since chondrogenesis was enhanced on large, sub-micron structures with native multilayers, while osteogenesis was improved on small, nanoscale structures with highly cross-linked multilayers. In conclusion, the novelty of this study lies in the combination of LIL with LbL. While LIL produces nanostructured surfaces of different dimensions with a low sample-to-sample variance (i), its combination with LbL allows for the modification with biomolecules, such as glycosaminoglycans, polypeptides and proteins (ii), but also for the control of viscoelastic properties in a spatial controlled manner (iii). This enables fundamental studies on cell adhesion and differentiation.

\section{Conflicts of interest}

There are no conflicts to declare.

\section{Acknowledgements}

The authors gratefully acknowledge the financial support of the German Research Foundation (DFG) through Grant GR 1290/10-1 and by the Fraunhofer Internal Programs (Grant No. Attract 069-608203). Further, Dr Bodo Fuhrmann, Dr Frank Heyroth, as well as Frank Syrowatka are acknowledged for their guidance during SEM and AFM measurements.

\section{References}

1 F. T. Bosman and I. Stamenkovic, J. Pathol., 2003, 200, 423428.

2 Kshitiz, J. Afzal, S. Y. Kim and D. H. Kim, Cell Adhes. Migr., 2015, 9, 300-307.

3 R. O. Hynes, Cell, 2002, 110, 673-687.

4 A. E. Aplin, A. Howe, S. K. Alahari and R. L. Juliano, Pharmacol. Rev., 1998, 50, 197-264.

5 F. G. Giancotti and E. Ruoslahti, Science, 1999, 285, 10281033.

6 Y. Liu, R. Medda, Z. Liu, K. Galior, K. Yehl, J. P. Spatz, E. A. Cavalcanti-Adam and K. Salaita, Nano Lett., 2014, 14, 5539-5546.

7 G. Blin, N. Lablack, M. Louis-Tisserand, C. Nicolas, C. Picart and M. Puceat, Biomaterials, 2010, 31, 1742-1750.

8 C. Zhao, A. Tan, G. Pastorin and H. K. Ho, Biotechnol. Adv., 2013, 31, 654-668.

9 P. Y. Wang, H. Thissen and P. Kingshott, Acta Biomater., 2016, 45, 31-59.

10 L. C. Kam, K. Shen and M. L. Dustin, Annu. Rev. Biomed. Eng., 2013, 15, 305-326.

11 J. M. Lee, A. A. Abdeen, A. S. Kim and K. A. Kilian, ACS Biomater. Sci. Eng., 2015, 1, 218-226.

12 D. Guduru, M. Niepel, J. Vogel and T. Groth, Int. J. Artif. Organs, 2011, 34, 963-985.

13 A. del Campo and E. Arzt, Chem. Rev., 2008, 108, 911-945.

14 Y. Xia and G. M. Whitesides, Annu. Rev. Mater. Sci., 1998, 28, 153-184. 
15 A. Köwitsch, M. Jurado Abreu, A. Chhalotre, M. Hielscher, S. Fischer, K. Mäder and T. Groth, Carbohydr. Polym., 2014, 114, 344-351.

16 A. del Campo and E. Arzt, Chem. Rev., 2008, 108, 911-945.

17 B. D. Gates, Q. B. Xu, M. Stewart, D. Ryan, C. G. Willson and G. M. Whitesides, Chem. Rev., 2005, 105, 11711196.

18 N. Roos, T. Luxbacher, T. Glinsner, K. Pfeiffer, H. Schulz and H.-C. Scheer, Proc. SPIE, 2001, 4343, DOI: 10.1117/ 12.436661 .

19 N. Faucheux, R. Schweiss, K. Lutzow, C. Werner and T. Groth, Biomaterials, 2004, 25, 2721-2730.

20 G. Krausch and R. Magerle, Adv. Mater., 2002, 14, 1579-1583.

21 M. Lazzari and M. A. Lopez-Quintela, Adv. Mater., 2003, 15, 1583-1594.

22 M. S. Niepel, B. Fuhrmann, H. S. Leipner and T. Groth, Langmuir, 2013, 29, 13278-13290.

23 G. Zhang and D. Wang, Chem. - Asian J., 2009, 4, 236-245.

24 B. K. Ekambaram, M. S. Niepel, B. Fuhrmann, G. Schmidt and T. Groth, ACS Biomater. Sci. Eng., 2018, 4(5), 18201832.

25 M. Ellman, A. Rodriguez, N. Perez, M. Echeverria, Y. K. Verevkin, C. S. Peng, T. Berthou, Z. Wang, S. M. Olaizola and I. Ayerdi, Appl. Surf. Sci., 2009, 255, 5537-5541.

26 A. Rodriguez, M. Echeverria, M. Ellman, N. Perez, Y. K. Verevkin, C. S. Peng, T. Berthou, Z. B. Wang, I. Ayerdi, J. Savall and S. M. Olaizola, Microelectron. Eng., 2009, 86, 937-940.

27 J. de Boor, N. Geyer, U. Gosele and V. Schmidt, Opt. Lett., 2009, 34, 1783-1785.

28 F. Y. Yu, P. Li, H. Shen, S. Mathur, C. M. Lehr, U. Bakowsky and F. Mucklich, Biomaterials, 2005, 26, 2307-2312.

29 G. Decher, Science, 1997, 277, 1232-1237.

30 M. Zhao, G. Altankov, U. Grabiec, M. Bennett, M. Salmeron-Sanchez, F. Dehghani and T. Groth, Acta Biomater., 2016, 41, 86-99.

31 J. J. Richardson, M. Bjornmalm and F. Caruso, Science, 2015, 348, aaa2491.

32 G. Decher and J. B. Schlenoff, in Multilayer Thin Films: Sequential Assembly of Nanocomposite Materials, ed. G. Decher and J. B. Schlenoff, Wiley-VCH, 2nd edn, 2012, DOI: 10.1002/9783527646746.ch1.

33 J. Borges and J. F. Mano, Chem. Rev., 2014, 114, 8883-8942.

34 M. Zhao, L. Li, C. Zhou, F. Heyroth, B. Fuhrmann, K. Maeder and T. Groth, Biomacromolecules, 2014, 15, 4272-4280.

35 N. Aggarwal, N. Altgarde, S. Svedhem, G. Michanetzis, Y. Missirlis and T. Groth, Macromol. Biosci., 2013, 13, 13271338.

36 J. M. Silva, A. R. Duarte, S. G. Caridade, C. Picart, R. L. Reis and J. F. Mano, Biomacromolecules, 2014, 15, 3817-3826.

37 M. S. Niepel, F. Almouhanna, B. K. Ekambaram, M. Menzel, A. Heilmann and T. Groth, Int. J. Artif. Organs, 2018, 41, 223-235.

38 L. Richert, F. Boulmedais, P. Lavalle, J. Mutterer, E. Ferreux, G. Decher, P. Schaaf, J. C. Voegel and C. Picart, Biomacromolecules, 2004, 5, 284-294.
39 J. H. Seo, J. H. Park, S. I. Kim, B. J. Park, Z. Ma, J. Choi and B. K. Ju, J. Nanosci. Nanotechnol., 2014, 14, 1521-1532.

40 C. Porcel, P. Lavalle, V. Ball, G. Decher, B. Senger, J.-C. Voegel and P. Schaaf, Langmuir, 2006, 22, 43764383.

41 M. S. Niepel, J. F. Mano and T. Groth, ACS Appl. Mater. Interfaces, 2016, 8, 25142-25151.

42 P. Kujawa, P. Moraille, J. Sanchez, A. Badia and F. M. Winnik, J. Am. Chem. Soc., 2005, 127, 9224-9234.

43 M. J. P. Biggs, R. G. Richards and M. J. Dalby, Nanomedicine, 2010, 6, 619-633.

44 S. G. Caridade, C. Monge, J. Almodovar, R. Guillot, J. Lavaud, V. Josserand, J. L. Coll, J. F. Mano and C. Picart, Acta Biomater., 2015, 15, 139-149.

45 A. B. D. Cassie and S. Baxter, Trans. Faraday Soc., 1944, 40, 546-551.

46 R. N. Wenzel, Ind. Eng. Chem., 1936, 28, 988-994.

47 R. McBeath, D. M. Pirone, C. M. Nelson, K. Bhadriraju and C. S. Chen, Dev. Cell, 2004, 6, 483-495.

48 D. Gugutkov, F. Awaja, K. Belemezova, M. Keremidarska, N. Krasteva, S. Kyurkchiev, G. Gallego-Ferrer, S. Seker, A. E. Elcin, Y. M. Elcin and G. Altankov, J. Biomed. Mater. Res., Part A, 2017, 105, 2065-2074.

49 C. H. Seo, K. Furukawa, K. Montagne, H. Jeong and T. Ushida, Biomaterials, 2011, 32, 9568-9575.

50 A. J. Engler, S. Sen, H. L. Sweeney and D. E. Discher, Cell, 2006, 126, 677-689.

51 R. Tzoneva, N. Faucheux and T. Groth, Biochim. Biophys. Acta, Gen. Subj., 2007, 1770, 1538-1547.

52 A. J. Engler, L. Richert, J. Y. Wong, C. Picart and D. E. Discher, Surf. Sci., 2004, 570, 142-154.

53 D. E. Discher, P. Janmey and Y. L. Wang, Science, 2005, 310, 1139-1143.

54 K. Burridge and M. Chrzanowska-Wodnicka, Annu. Rev. Cell Dev. Biol., 1996, 12, 463-518.

55 S. Hehlgans, M. Haase and N. Cordes, Biochim. Biophys. Acta, Rev. Cancer, 2007, 1775, 163-180.

56 R. M. Salasznyk, R. F. Klees, W. A. Williams, A. Boskey and G. E. Plopper, Exp. Cell Res., 2007, 313, 22-37.

57 C. Moerke, P. Mueller and J. B. Nebe, J. Mater. Sci.: Mater. Med., 2017, 28, 171.

58 B. Y. Xu, G. B. Song, Y. Ju, X. Li, Y. H. Song and S. Watanabe, J. Cell. Physiol., 2012, 227, 2722-2729.

59 L. P. Xu, J. Meng, S. Zhang, X. Ma and S. Wang, Nanoscale, 2016, 8, 12540-12543.

60 S. Mechiche Alami, S. C. Gangloff, D. Laurent-Maquin, Y. Wang and H. Kerdjoudj, Stem Cells Transl. Med., 2016, 5, 1587-1593.

61 J. A. Panadero, S. Lanceros-Mendez and J. L. Ribelles, Acta Biomater., 2016, 33, 1-12.

62 J. Zhang, Y. Wu, T. Thote, E. H. Lee, Z. Ge and Z. Yang, Biomed. Mater., 2014, 9, 035011.

63 H. Nobusue, N. Onishi, T. Shimizu, E. Sugihara, Y. Oki, Y. Sumikawa, T. Chiyoda, K. Akashi, H. Saya and K. Kano, Nat. Commun., 2014, 5, 3368. 